\title{
Quantification of myocardial deformation in patients with Fabry disease by cardiovascular magnetic resonance feature tracking imaging
}

\author{
Lei Zhao ${ }^{1}$, Chen Zhang ${ }^{1}$, Jie Tian ${ }^{1}$, Madiha Saiedi ${ }^{2}$, Chenyao $\mathrm{Ma}^{3}$, Ning Li ${ }^{1}$, Fang Fang ${ }^{3}$, Xiaohai Ma \\ Joseph Selvanayagam ${ }^{2}$
}

${ }^{1}$ Department of Radiology, Beijing Anzhen Hospital, Capital Medical University, Beijing, China; ${ }^{2}$ College of Medicine and Public Health, Flinders University of South Australia, Adelaide, Australia; ${ }^{3}$ Department of Sleep Medical Center, Beijing Anzhen Hospital, Capital Medical University, Beijing, China; ${ }^{4}$ Department of Interventional Therapy, Beijing Anzhen Hospital, Capital Medical University, Beijing, China

Contributions: (I) Conception and design: L Zhao, X Ma; (II) Administrative support: X Ma; (III) Provision of study materials or patients: F Fang, C Ma; (IV) Collection and assembly of data: C Zhang, J Tian, N Li; (V) Data analysis and interpretation: L Zhao, C Zhang, J Tian, N Li; (VI) Manuscript writing: All authors; (VII) Final approval of manuscript: All authors.

Correspondence to: Xiaohai Ma. No. 2 Anzhen Rd., Chaoyang Dist., Beijing, China. Email: maxi8238@yahoo.com.

Background: Cardiac involvement is a major contributor of morbidity and mortality in Fabry disease (FD). Early detection and accurate evaluation of the disease progression is important in management. Cardiovascular magnetic resonance (CMR) derived feature fracking (FT) is a validated quantitative method of assessing myocardial deformation which may reflect early changes of myocardial function and track disease severity. We sought to evaluate the utility of CMR-FT as a measure of myocardial dysfunction in FD.

Methods: Twenty FD patients (12 males, 40.8 \pm 14.9 years) and 20 age and sex matched healthy controls (10 males, 40.5 \pm .2 years) were prospectively enrolled. Subjects underwent CMR including cine, pre-/postcontrast T1 mapping and late gadolinium enhancement (LGE). FD patients were divided into three groups; group 1: patients without left ventricular hypertrophy (LVH) and LGE negative; group 2: patients with LVH positive, LGE either positive or negative; group 3: patients with LGE positive, LV wall thinning and heart failure. FT derived strain indices were measured and its associations with other processes were investigated.

Results: In FD patients, 14 (70\%) had LVH and 4 (20\%) had LGE. Compared with normal controls, LV global longitudinal strain (GLS) were reduced significantly in all three Fabry groups (all $\mathrm{P}<0.05)$, global circumferential strain (GCS) were reduced only in group 2 and group $3(\mathrm{P}<0.05)$. Among three FD groups, there were significant differences of LV GLS, GCS, native T1 value and extracellular volume fraction (ECV) (all $\mathrm{P}<0.01$ ), group 1 had mild LV strain indices impairment, group 3 had the most severe LV strain indices. When compared between FD subgroups, GLS and GCS showed significant difference between each two groups (all $\mathrm{P}<0.05$ ). There were weak correlations between the $\mathrm{LV}$ functional parameters (ejection fraction, LV mass index), maximal wall thickness, T1 mapping indices (native T1, ECV) and LV strain indices. The strongest relation was between global longitudinal early diastolic strain rate and native $\mathrm{T} 1$ value $(\mathrm{r}=0.783$, $\mathrm{P}<0.01)$.

Conclusions: CMR strain imaging identifies myocardial deformation in FD in different stages. Strain imaging can track disease severity and may be an alternative method for follow-up of FD patients.

Keywords: Fabry disease (FD); strain indices; T1 mapping; stage

Submitted Nov 01, 2020. Accepted for publication Dec 28, 2020.

doi: $10.21037 / \mathrm{cdt}-20-897$

View this article at: http://dx.doi.org/10.21037/cdt-20-897 


\section{Introduction}

Fabry disease (FD) is a rare $\mathrm{X}$-linked lysosomal storage disorder caused by mutations in the gene encoding for $\alpha$-galactosidase A (1). FD affects multiple organs including the heart. Cardiac involvement is a major contributor of morbidity and mortality in FD $(2,3)$. Detection of early involvement, assessment of cardiac response to treatment and evaluation of disease severity are important in FD management $(4,5)$. Cardiovascular magnetic resonance $(\mathrm{CMR})$ is a non-invasive and multi-parametric imaging modality that has the potential to detect early cardiac involvement in FD and has quickly become the referencestandard imaging method. CMR allows for a detailed assessment of regional myocardial wall thickness, myocardial mass, myocardial fat (using native T1 mapping technique to reflect sphingolipid storage), fibrosis [using the late gadolinium enhancement (LGE) technique]. In the early stage of FD, even in the absence of left ventricular hypertrophy $(\mathrm{LVH})$, low native $\mathrm{T} 1$ values were found to sensitively indicate sphingolipid accumulation (6). When patients progress to myocardial fibrosis or triggered $\mathrm{LVH}$ in men, native $\mathrm{T} 1$ values increase and may result in "pseudonormalization" $(7,8)$. CMR derived feature fracking (FT) is a validated quantitative method of assessing myocardial deformation which may reflect early changes of myocardial structure and function in many diseases (9). Therefore, we sought to explore the utility of CMR-FT as a measure of myocardial dysfunction in FD patients with or without LVH and/or fibrosis. We present the following article in accordance with the Materials Design Analysis Reporting (MDAR) checklist. Available at: http://dx.doi. org/10.21037/cdt-20-897.

\section{Methods}

The study was conducted in accordance with the Declaration of Helsinki (as revised in 2013). The study was approved by Beijing Anzhen Hospital ethics committee (NO.: 2019065X) and informed consent was taken from all the patients.

\section{Study populations}

Twenty patients with genetically confirmed FD were prospectively enrolled. None of the patients with FD had received enzyme replacement therapy prior to the study entry. Exclusion criteria included prior myocardial infarction or known coronary artery disease and contraindications to CMR. Twenty healthy volunteers without history of cardiovascular disease were also enrolled as a control group.

\section{CMR image acquisition}

CMR was performed using a 3T MR system (MAGNETOM Verio, Siemens Healthcare, Erlangen, Germany) with a 32-channel cardiac coil. Steady-state free-precession cine images were obtained during repeated breath-holds in three long axes (2-chamber, 3-chamber, and 4-chamber view) and in a stack of short axes covering the LV. Imaging parameters were: repetition time (TR) $3.1 \mathrm{~ms}$, echo time (TE) $1.3 \mathrm{~ms}$, asymmetric echo with factor 0.29 , flip angle (FA) $45^{\circ}$, field of view (FOV) $276 \times 340 \mathrm{~mm}^{2}$, matrix 156×192, slice thickness 6 mm, receiver bandwidth (BW) $704 \mathrm{~Hz} / \mathrm{px}$, parallel imaging using GRAPPA reconstruction $(\mathrm{R}=2), 30$ cardiac phases. $\mathrm{LV}$ native $\mathrm{T} 1$ maps and 20 min post-contrast $\mathrm{T} 1$ maps were acquired using modified Look-Locker inversion recovery sequence [MOLLI, sampling pattern: 5(3)3] at basal, midventricular and apical short axis slices. Imaging parameters were: $\mathrm{TR}=2.6-2.7 \mathrm{~ms}, \mathrm{TE}=1.0-1.1 \mathrm{~ms}, \mathrm{FA}=35^{\circ}$, FOV $=270 \times 360 \mathrm{~mm}^{2}$, matrix $=256 \times 256$, slice thickness $=6 \mathrm{~mm}$, $\mathrm{BW}=1,045-1,028 \mathrm{~Hz} / \mathrm{px}$, GRAPPA acceleration factor 2, linear phase-encoding ordering. Quality control was performed during scanning by reviewing the "goodness of fit" map and source images to allow an immediate repetition of suboptimal measurements to minimize the respiratory motion and offresonance effects. Extracellular volume fraction (ECV) was derived from the pre-/post-contrast T1 maps and hematocrit. The blood sample was taken just before the CMR exam to measure hematocrit for ECV calculation. A commercially available gadolinium-based contrast agent (gadopentetate dimeglumine, Magnevist, Bayer Healthcare) was administered intravenously at a dose of $0.1 \mathrm{mmol} / \mathrm{kg}$ body weight and contrast-enhanced images were acquired after a 10-min delay with the use of an inversion-recovery segmented gradient echo sequence. Contrast-enhanced images were acquired in identical long- and short-axis planes to the cine images except for the most apical short-axis slice, which was excluded because it can be affected by partial-volume effects. The inversion time was meticulously adjusted to obtain maximal nulling of remote normal $\mathrm{LV}$ myocardium with voxel sizes of $1.9 \mathrm{~mm}$ $\times 1.4 \mathrm{~mm} \times 7.0 \mathrm{~mm}$.

\section{CMR analysis}

The LV functional indices and maximum wall thickness (MWT) of all subjects were analyzed using dedicated 
software (Argus, Siemens Healthcare, Erlangen, Germany). The following indices were measured: $L V$ ejection fraction (LVEF), LV end-diastolic volume index (LVEDVi), LV endsystolic volume index (LVESVi), LV cardiac output (LVCO) and $L V$ mass index (LVMi). LV wall thickness was measured at end-diastolic phase on the short-axis images with American Heart Association (AHA) 16-segmental mode, a MWT $>12 \mathrm{~mm}$ or LVMi $>85 \mathrm{~g} / \mathrm{m}^{2}$ in males and $>81 \mathrm{~g} / \mathrm{m}^{2}$ in females were considered as being LVH positive $(10,11)$. All LGE images were visually assessed for the presence of LGE. Quantitative LGE analysis was performed using a signal intensity threshold of 4 standard deviations (SD) above visually normal remote myocardium, expressed as absolute myocardial mass gram (12). Analysis of 3-dimensional (3D) LV strain and strain rate were obtained using commercially available software as previously described (13) (CVi42 version 5.9.1, Circle, Canada). Epicardial and endocardial borders of LV were manually drawn on the end-diastolic frame of short axis and long axis images respectively. Then the contours were automatically propagated in all frames throughout the entire cardiac cycle. CMR derived FT was visually reviewed to ensure accurate tracking. In cases of inadequate tracking, the myocardial borders were manually adjusted. LV global peak systolic longitudinal (GLS), circumferential (GCS) strain, LV global peak systolic longitudinal (GLSR), circumferential (GCSR) strain rates, LV global peak early diastolic longitudinal (GDLSR) and circumferential (GDCSR) strain rates were all derived. LV native $T 1$ values and post-contrast $T 1$ values were measured with AHA 16-segmental mode, global native T1 values and post-contrast $\mathrm{T} 1$ values were obtained by averaging segmental values respectively. Accordingly, segmental and global ECV were obtained (8).

The intra- and inter-observer variability for the LV strain indices were assessed by the intraclass correlation coefficient (ICC) in all patients. Intra-observer reproducibility was established by the same observer who re-analyzed all patients' images after one month. Inter-observer reproducibility was assessed by a second-independent observer blinded to the first observer's results.

\section{Subgroup analysis}

Patients were divided into three groups based on their clinical assessment of heart failure, blood biomarkers (troponin, brain natriuretic peptide), cardiac functional indices (by echocardiography or CMR), electrocardiography and CMR findings (14-16): group 1: patients without LVH,
LGE negative, and without heart failure; group 2: patients with LVH positive, LGE either positive or negative, and without heart failure; group 3: patients with LGE positive, $\mathrm{LV}$ wall thinning compared to normal controls, presence of conduction abnormalities and heart failure (Figure 1).

\section{Statistical analysis}

Statistical analyses were carried out using SPSS (version 22, $\mathrm{IBM})$. Continuous variables are expressed as mean $\pm \mathrm{SD}$ and categorical as frequencies or percentages. Normality was checked using the Shapiro-Wilk test. Differences between FD patients and healthy controls were compared using the paired $t$-test or $\mathrm{U}$ test or $\chi^{2}$ testing as indicated. Differences across three FD groups were compared with using ANOVA or Kruskal-Wallis for parametric and nonparametric variables, respectively, and $\chi^{2}$ testing for categorical variables. Person's or Spearman's correlation was performed to investigate the potential relationship between strain indices and other CMR indices (including LV functional indices, MWT, native T1, ECV) in all patients. The correlation was considered weak if correlation coefficient was $<0.5$, moderate if correlation coefficient was between $0.5-0.7$, and strong if correlation coefficient was $>0.7$. A $\mathrm{P}<0.05$ was considered significant.

\section{Results}

\section{Baseline clinical characteristics of FD patients}

Baseline demographic data and CMR findings of all subjects are summarized in Table 1. There were no statistical differences of age, sex, height and weight between FD patients and healthy controls (all $\mathrm{P}>0.05$ ). Age and sex between FD patients and healthy controls also showed no statistical differences in every subgroup (Table 2). Among FD patients, 7 (35\%) patients had a history of hypertension and $1(5 \%)$ patient had a history of diabetes mellitus, 14 (70\%) had LVH (mean MWT of these 14 patients was $15.2 \pm 2.0 \mathrm{~mm})$ and $4(20 \%)$ had LGE. Phenotypes and the enzymatic activity of FD patients are summarized in Table 3.

\section{$L V H$ and $L V$ global function findings of FD patients according to group}

There were statistical differences of age among FD patients $(\mathrm{P}=0.006)$, group 3 of $\mathrm{FD}$ patients had significant older mean age than other FD groups (all $\mathrm{P}<0.05$ ). The MWT, LVEF 

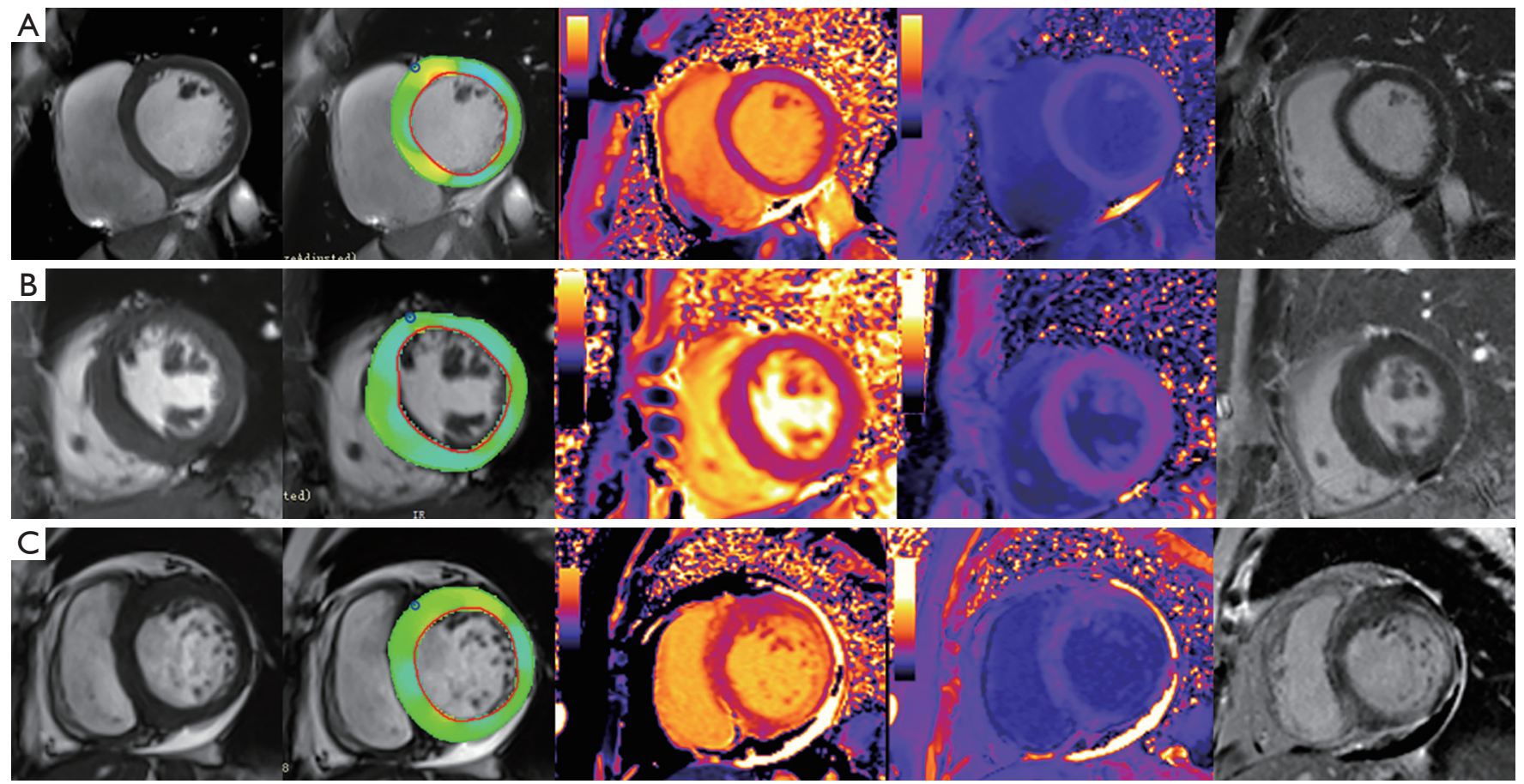

Figure 1 Examples of four Fabry disease groups. (A) Example of group 1, LVEF =63\%, no LVH, mean LV native T1 =1,129.7 ms, mean $\mathrm{ECV}=24.1 \%$, no LGE. (B) Example of group 2, LVEF $=74 \%$, had LVH, mean LV native T1 =1,153.5 ms, mean ECV =23.3\%, no LGE. (C) Example of group 3, LVEF =44\%, LV inferior-lateral wall thinning, mean LV native T1 =1,343.1 ms, mean ECV =33.4\%, LV extensive LGE, had pericardial effusion. First column: cine images; second column: strain analysis images; third column: native T1 maps (normal range of T1: 1,212.8-1,265.0 ms); fourth column: post-contrast T1 maps; fifth column: LGE images. LVEF, left ventricular ejection fraction; $\mathrm{LVH}$, left ventricular hypertrophy; ECV, extracellular volume fraction; LGE, late gadolinium enhancement.

and LVMi were significantly different among three FD groups (all $\mathrm{P}<0.01$ ) and between each two groups (all $\mathrm{P}<0.01$ ) except MWT between group 2 and group 3 ( $\mathrm{P}=0.071)$, LVEF between group 1 and group 2 (P=0.274). The MWT, LVEF and LVMi were significantly different between FD patients and sex and age matched healthy controls in group 2 and group 3 respectively (all $\mathrm{P}<0.01$, Table 2 ).

\section{Myocardial deformation imaging findings of FD patients according to group}

Compared with healthy controls, all the strain indices of FD with LVH (group 2 and group 3) were reduced (all $\mathrm{P}<0.05$ ) except GCSR in group $2(\mathrm{P}=0.932)$, but in FD without LVH (group 1), only GLS and GDLSR were significantly lower than that of controls (all $\mathrm{P}<0.05$, Table 2). Among FD groups, the strain indices impairment degree from mild to severe were group $1<$ group $2<$ group 3 . When compared between different FD groups, there were significant differences of GLS and GCS among three groups and between each two groups (Figure 2). There were weak correlations between the LV functional parameters (LVEF, LVMi), MWT, T1 mapping indices (native T1, ECV) and LV strain indices (GLS, GLSR, GDLSR, GCS, GCSR, GDCSR) (Table 4). The strongest relation was between GDLSR and native $\mathrm{T} 1$ value $(\mathrm{r}=0.783, \mathrm{P}<0.01)$.

The strain indices were reproducible on an intra- and inter-observer level. For intra-observer reproducibility, ICC of GCS was 0.854 , GCSR was 0.806 , GDCSR was 0.807 , ICC of GLS was 0.799 , GLSR was 0.756 , GDLSR was 0.749. For inter-observer reproducibility, ICC of GCS was 0.818 , GCSR was 0.781 , GDCSR was 0.763 , ICC of GLS was 0.725 , GLSR was 0.698 , GDLSR was 0.598 .

\section{Tissue characterization findings of FD patients according to group}

Overall mean LV native T1 value of FD $(1,160.4 \pm 81.2 \mathrm{~ms})$ 
Table 1 Demographic data and CMR findings of Fabry disease patients and healthy controls

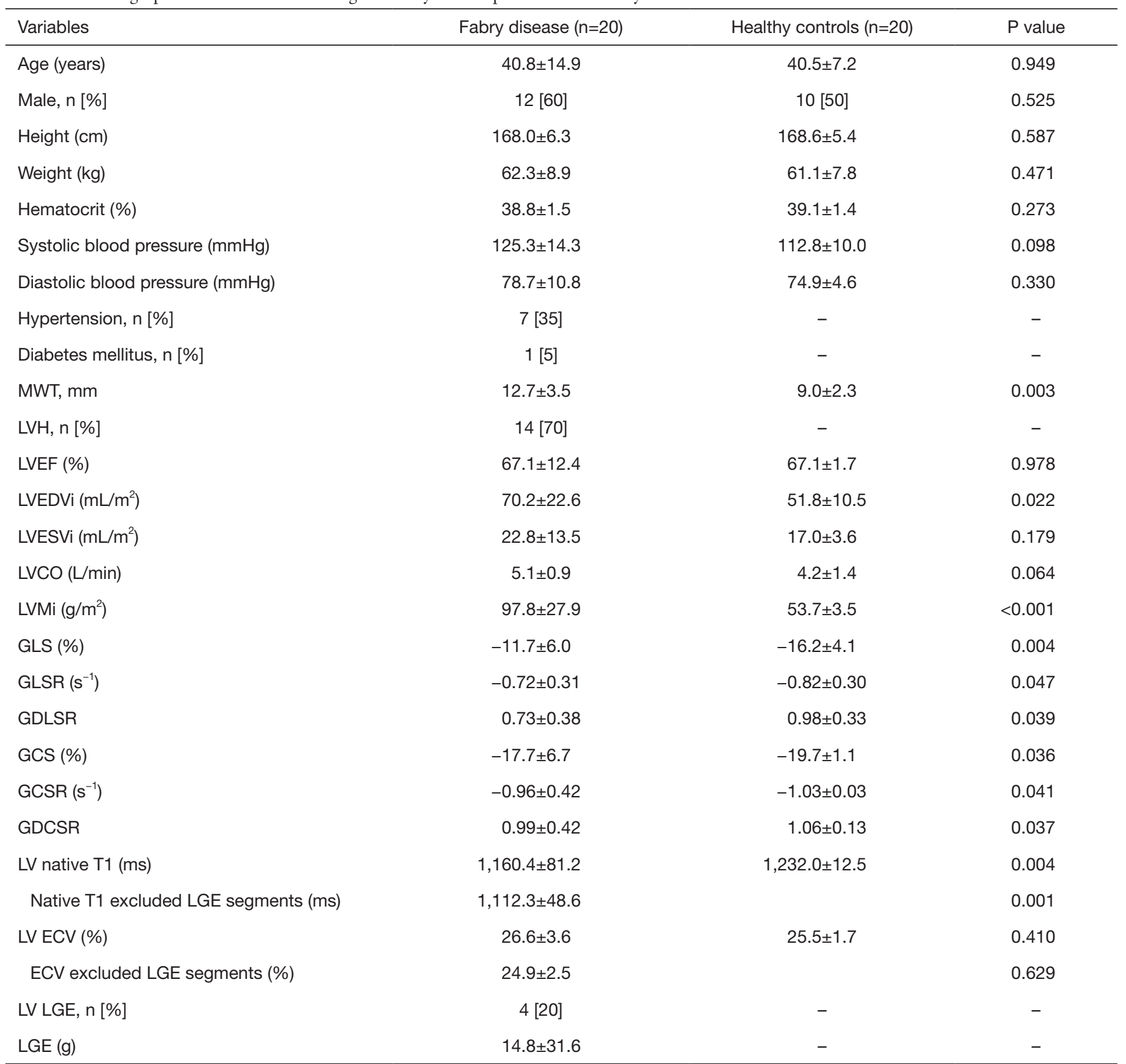

Data are expressed as mean \pm SD, or as $n[\%]$. CMR, cardiovascular magnetic resonance; ECV, extracellular volume fraction; GCS, global peak systolic circumferential strain; GCSR, global peak systolic circumferential strain rates; GDCSR, global peak early diastolic circumferential strain rate; GDLSR, global peak early diastolic longitudinal strain rate; GLS, global peak systolic longitudinal strain; GLSR, global peak systolic longitudinal strain rate; LGE, late gadolinium enhancement; LVCO, left ventricular cardiac output; LVEDVi, left ventricular end-diastolic volume index; LVEF, left ventricular ejection fraction; LVESVi, left ventricular end-systolic volume index; LVH, left ventricular hypertrophy; LVMi, left ventricular mass index; MWT, maximal wall thickness. 


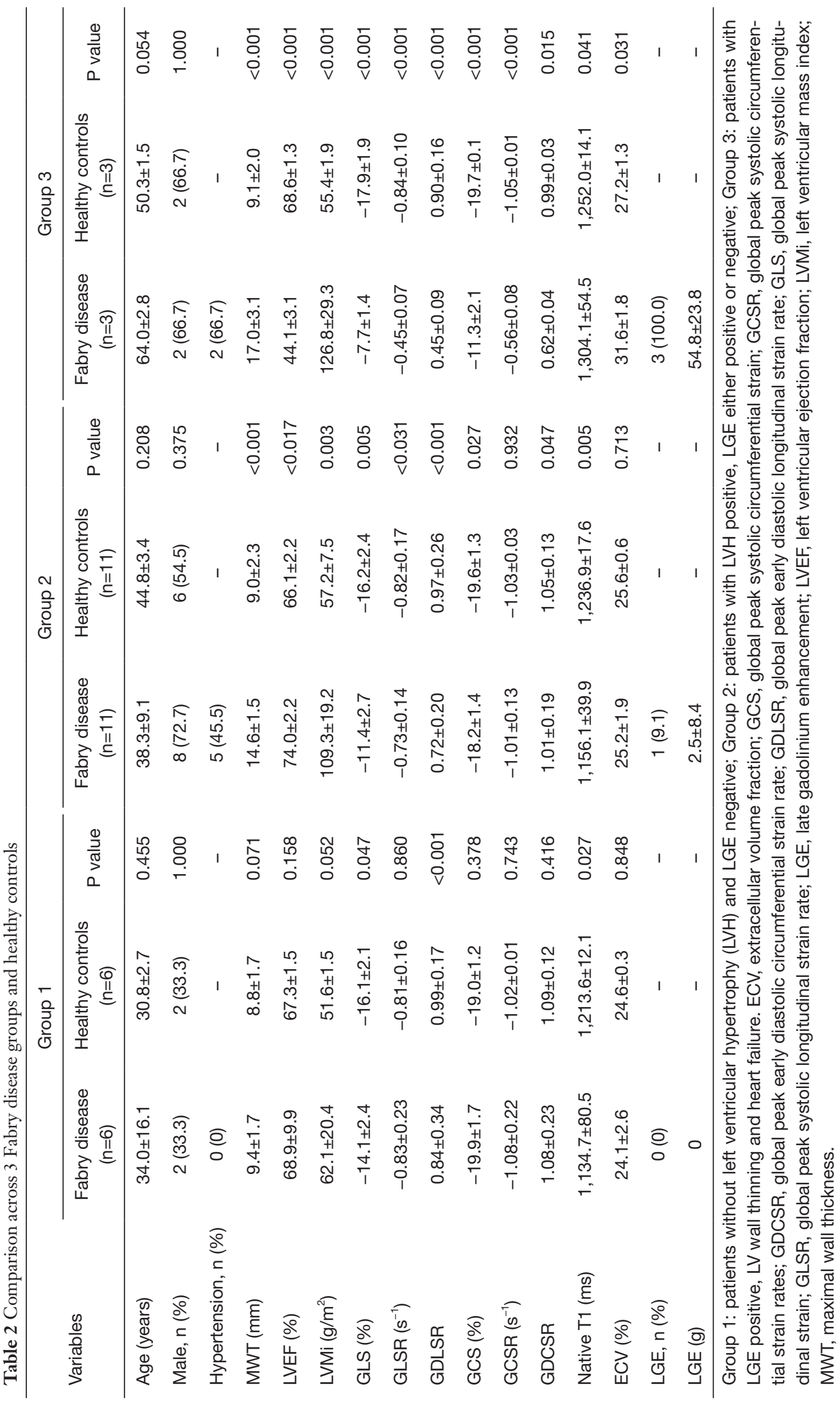


Table 3 Phenotypes and the enzymatic activity of Fabry disease patients

\begin{tabular}{lcc}
\hline Phenotypes & Male patients $(\mathrm{n}=12)$ & Female patients $(\mathrm{n}=8)$ \\
\hline Classic phenotype patients & 10 & 2 \\
Average age (min-max) & $32.4(15.0-36.0)$ & $38.0(37.0-39.0)$ \\
Average $\alpha$-gal A activity (min-max) & $0.6(0-3.2)$ & $13.3(11.2-26.6)$ \\
Late-onset patients & 2 & 6 \\
Average age (min-max) & $51.5(41.0-62.0)$ & $51.7(44.0-66.0)$ \\
Average $\alpha$-gal A activity (min-max) & $3.0(0.9-8.1)$ & $16.5(10.8-21.9)$ \\
\hline
\end{tabular}

Enzyme activity is measured in $\mathrm{nmol} / \mathrm{g} / \mathrm{min}$; normal range $=24.5-63.6 \mathrm{nmol} / \mathrm{g} / \mathrm{min}$.

Table 4 Correlation of LV strain indices and other CMR parameters in the Fabry disease patients

\begin{tabular}{lcccccc}
\hline Parameters & GLS & GLSR & GDLSR & GCS & GCSR \\
\hline LVEF & $-0.621^{*}$ & $-0.640^{*}$ & $0.646^{*}$ & $-0.767^{\star *}$ & -0.532 & 0.529 \\
LVMi & 0.220 & $0.616^{*}$ & -0.579 & 0.197 & 0.435 \\
MWT & 0.402 & 0.342 & -0.526 & 0.279 & 0.230 \\
Native T1 & 0.515 & $0.648^{*}$ & $-0.783^{\star *}$ & 0.526 & -0.546 \\
ECV & 0.366 & $0.753^{*}$ & -0.437 & 0.443 & 0.427 & $0.789^{\star}$ \\
\hline
\end{tabular}

Data represents the correlation coefficients. *, $\mathrm{P}<0.05$; ${ }^{*}, \mathrm{P}<0.01$. CMR, cardiovascular magnetic resonance; GLS, global peak systolic longitudinal strain; GLSR, global peak systolic longitudinal strain rate; GDLSR, global peak early diastolic longitudinal strain rate; GCS, global peak systolic circumferential strain; GCSR, global peak systolic circumferential strain rates; GDCSR, global peak early diastolic circumferential strain rate; LVEF, left ventricular ejection fraction; LVMi, left ventricular mass index; MWT, maximal wall thickness; ECV, extracellular volume fraction.

was lower than that of controls $(1,232.0 \pm 12.5 \mathrm{~ms}, \mathrm{P}<0.01)$, after excluded LGE positive segments, mean LV native T1 value of $1,112.3 \pm 48.6 \mathrm{~ms}$ was further lower compared to healthy controls (Table 1). There were statistical differences of $\mathrm{LV}$ native $\mathrm{T} 1$ values between FD patients and age and sex matched healthy controls (Table 2). When compared between different FD groups, FD without LVH (group 1) had lower native T1 values and ECV than FD with $\mathrm{LVH}$ (group 2 and group 3), but the differences were not significant when compared to group 2 (all $\mathrm{P}>0.05$ ) (Figure 2).

\section{Discussion}

In this study we examined the value of CMR-FT in the assessment of myocardial deformation in patients with FD. The principal findings of our study are: (I) impaired LV strain indices occur in FD patients at all stages of the disease. The impairment severity in myocardial deformation parallels along with disease severity. (II) In the earlier stage of the disease (without LVH), both
LV systolic (global peak systolic longitudinal strain) and diastolic (early diastolic longitudinal strain rate) myocardial contractility were impaired compared with the normal controls. LV longitudinal strain indices are more sensitive than circumferential strain indices in detection of the early contractile abnormality in FD. (III) Also, in the earlier stages of the disease, although $L V$ native $T 1$ values overlap between patients with $\mathrm{LVH}$ and without $\mathrm{LVH}, \mathrm{LV}$ strain indices in FD patients with LVH were significantly more impaired when compared to FD patients without LVH. Therefore, the distinctive $\mathrm{T} 1$ reduction makes native $\mathrm{T} 1$ mapping a valuable screening tool for $\mathrm{FD}$, however, CMRFT strain appears to be appropriate for tracking disease severity in FD.

CMR myocardial feature tracking is a new technique that allows for precise measurement of both global and regional myocardial strain. CMR-FT has been validated against traditional tagging methods of measuring strain and has also been able to distinguish between myocardial deformations in cardiac diseases including hypertrophic 

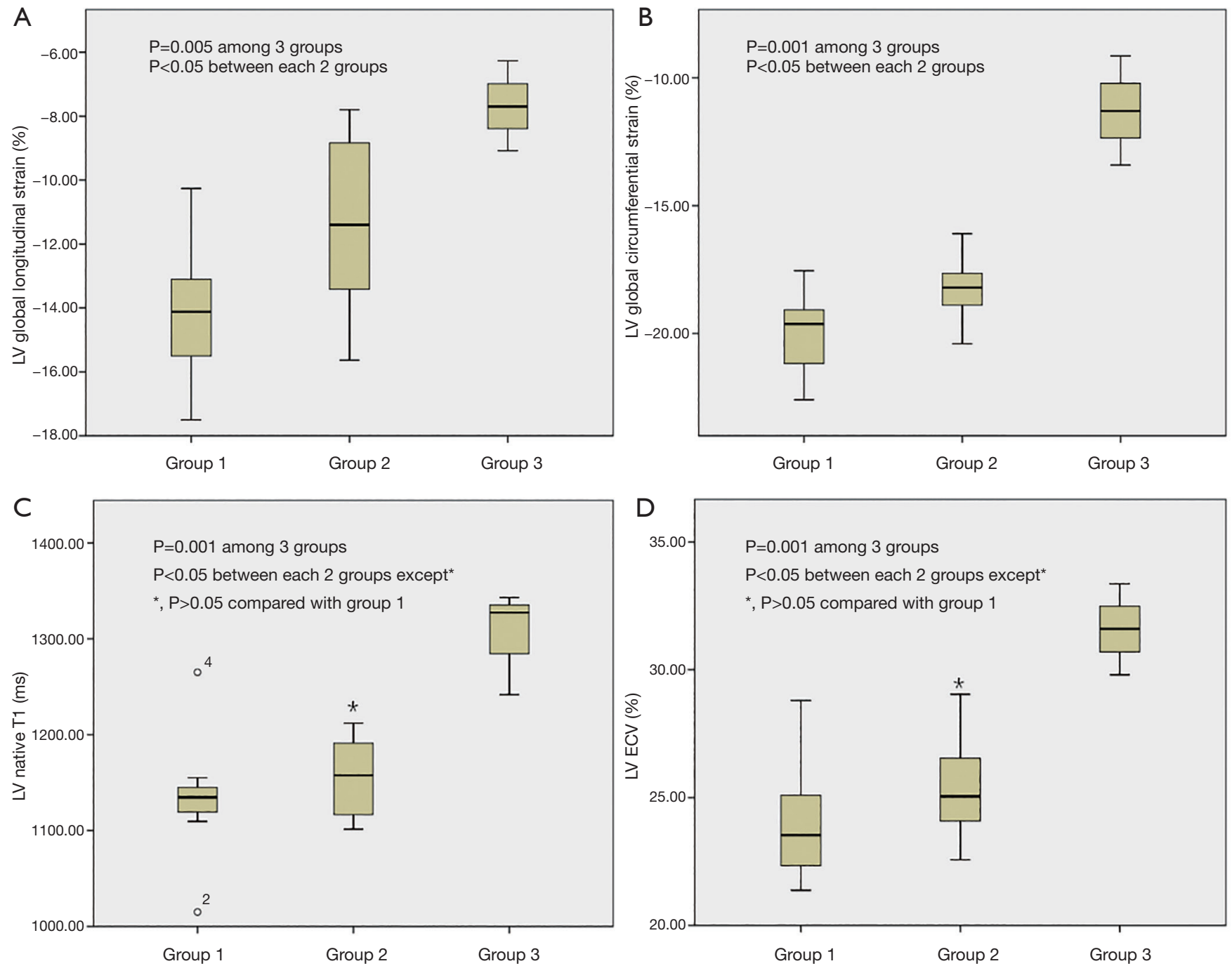

Figure 2 Box plots comparing strain indices and T1 mapping values between different Fabry disease stage groups. (A) LV global longitudinal strain was significantly different among three Fabry disease groups $(\mathrm{P}=0.005)$ and between each two groups (all $\mathrm{P}<0.05)$. (B) LV global circumferential strain was significantly different among three Fabry disease groups $(\mathrm{P}=0.001)$ and between each two groups $($ all $\mathrm{P}<0.05)$. (C) LV native T1 value was significantly different among three Fabry disease groups $(\mathrm{P}=0.001)$, but showed no significant difference between group 1 and group 2 ( $\left.^{*}, \mathrm{P}=0.461\right)$. (D) LV ECV was significantly different among three Fabry disease groups (P=0.001) but showed no significant difference between group 1 and group $2\left(^{*}, \mathrm{P}=0.340\right)$.

cardiomyopathy (HCM), cardiac amyloidosis and myocarditis (17-20). Abnormal strain values have previously been reported in FD patients with $\mathrm{LVH}$ in few studies using both echocardiographic strain and CMR feature tracking $(10,12,21,22)$. The results in our study extends these prior findings and suggest that the degree of strain abnormalities varied significantly in all three FD groups and correlated with disease severity. Myocardial inflammation, hypertrophy and fibrosis follow sphingolipid deposition in
FD. Myocardial inflammation, hypertrophy and fibrosis all have an adverse impact on myocardial contractility and lead to reduced myocardial strain indices in many cardiovascular diseases (23-25). In our study, group 1 only had mild LV native $\mathrm{T} 1$ reduction and mild myocardial strain indices impairment; group 2, in addition to $\mathrm{LV}$ native $\mathrm{T} 1$ reduction, this group also had LVH and myocardial fibrosis (assessed by LGE), correspondingly worse myocardial strain indices; and as a late stage, group 3 had LVH, heavy myocardial fibrosis 
burden, heart failure, and the worst myocardial strain indices. Therefore, CMR-FT may hold promise in the monitoring of FD cardiomyopathy progression in patients with FD.

Early detection of cardiac involvement even in lateonset FD allows for appropriate treatment decisions (26). Although myocardial strain abnormalities have previously been demonstrated in FD patients with LVH and without $\mathrm{LVH}$, there has been contradictory findings in prior studies. Mathur et al. found that FD patients without LVH compared with controls did not differ significantly in GLS or GCS (12). Another group assessed GLS by CMR and echocardiography in serial studies and showed that FD patients without LVH had impaired GLS $(10,27,28)$. In this present study, we found systolic and diastolic longitudinal strain indices, global peak systolic longitudinal strain and early diastolic longitudinal strain rate respectively were lower than normal in FD patients without LVH. However, circumferential strain indices were not different compared with healthy controls. These results suggested that the myocardial mechanical impairment assessed by GLS is sensitive in detecting the early contractile abnormality. Knott et al. assessed myocardial blood flow in FD patients and found impaired myocardial perfusion even before the onset of LVH. Their results also demonstrated that the FD perfusion impairment was more pronounced in the subendocardium (8). The subendocardium of LV is longitudinal oriented fibres and the abnormality of perfusion in the subendocardium may partially explain the GLS reduction in the early stage of the disease.

Low native T1 can detect cardiac involvement in FD as it represents sphingolipid accumulation $(27,29)$. Our results showed that average $L V$ native $T 1$ values even in patients without LVH (early stage) were lower than normal controls, which is in line with previous studies $(7,8,10)$. Notably, $\mathrm{LV}$ native $\mathrm{T} 1$ values in the early stage without $\mathrm{LVH}$ was even lower than that in patients in the intermediate stage with LVH. Furthermore, in the late stage, average native $\mathrm{T} 1$ was significantly higher than normal values. Reduction of native T1 values did not track disease severity. Native T1 reflects the overall myocardial changes including the myocyte and interstitium changes. Multiple pathology of FD, such as edema, fibrosis, and LVH lead to native $\mathrm{T} 1$ pseudonormalization by increasing the $\mathrm{T} 1$ values (7). Therefore, native $\mathrm{T} 1$ is sensitive for screening cardiac involvement (14), but when using native $\mathrm{T} 1$ for followup treatment, the changes of $\mathrm{T} 1$ (either progression or regression) may need cautious interpretation due to the impact of above factors. Previous studies have shown that the myocardial ECV in FD is similar to healthy controls which our results agree with for the early and intermediate stages. Furthermore, like other cardiomyopathies in the late stage, LV ECV in FD was elevated significantly in the final stage. In FD, ECV provided similar clinical significance like most cardiovascular diseases.

\section{Limitations}

There are several limitations to our study. FD is a rare disease, thus limiting the number of patients included in the study. We did not undertake myocardial biopsies in the patients to determine the lysosomal storage and myocardial fibrosis. Given the small number of patients, we were not able to compare the CMR indices between male and female in different stages. The values of myocardial strain assessed by 3D FT-CMR were lower than 2D FT-CMR (30). We used a 3D strain method, so the strain values of this study have to be interpreted within the same assessment method. Hypertension is one of the main adverse events occurring in the late stage of FD (31). We have seven patients with hypertension (five patients in group 2, two patients in group 3), LV hypertrophy, myocardial deformation and fibrosis in these patients maybe partially explained by hypertension. Future investigations are needed to investigate the effects of hypertension on the LV morphology, function and tissue characterization in FD.

\section{Conclusions}

Myocardial strain impairment is found in FD in all stages after diagnosis, even in the early stage, without LVH. CMR derived strain imaging may aid in the early diagnosis and appropriate management of patients with cardiac FD.

\section{Acknowledgments}

Funding: This work was supported by the National Natural Science Foundation of China (grant number 81671647, 81771787).

\section{Footnote}

Reporting Checklist: The authors have completed the MDAR reporting checklist. Available at http://dx.doi.org/10.21037/ cdt-20-897

Data Sharing Statement: Available at http://dx.doi. org/10.21037/cdt-20-897 
Peer Review File: Available at http://dx.doi.org/10.21037/ cdt-20-897

Conflicts of Interest: All authors have completed the ICMJE uniform disclosure form (available at http://dx.doi. org/10.21037/cdt-20-897). The authors have no conflicts of interest to declare.

Ethical Statement: The authors are accountable for all aspects of the work in ensuring that questions related to the accuracy or integrity of any part of the work are appropriately investigated and resolved. The study was conducted in accordance with the Declaration of Helsinki (as revised in 2013). The study was approved by Beijing Anzhen Hospital ethics committee (NO.: 2019065X) and informed consent was taken from all the patients.

Open Access Statement: This is an Open Access article distributed in accordance with the Creative Commons Attribution-NonCommercial-NoDerivs 4.0 International License (CC BY-NC-ND 4.0), which permits the noncommercial replication and distribution of the article with the strict proviso that no changes or edits are made and the original work is properly cited (including links to both the formal publication through the relevant DOI and the license). See: https://creativecommons.org/licenses/by-nc-nd/4.0/.

\section{References}

1. Zarate YA, Hopkin RJ. Fabry's disease. Lancet 2008;372:1427-35.

2. Waldek S, Patel MR, Banikazemi M, et al. Life expectancy and cause of death in males and females with Fabry disease: findings from the Fabry Registry. Genet Med 2009;11:790-6.

3. Patel MR, Cecchi F, Cizmarik M, et al. Cardiovascular events in patients with fabry disease natural history data from the fabry registry. J Am Coll Cardiol 2011;57:1093-9.

4. Arends M, Biegstraaten M, Hughes DA, et al. Retrospective study of long-term outcomes of enzyme replacement therapy in Fabry disease: analysis of prognostic factors. PLoS One 2017;12:e0182379.

5. Hanneman K, Karur GR, Wasim S, et al. Prognostic significance of cardiac magnetic resonance imaging late gadolinium enhancement in Fabry disease. Circulation 2018;138:2579-81.

6. Nordin S, Kozor R, Baig S, et al. Cardiac phenotype of prehypertrophic fabry disease. Circ Cardiovasc Imaging 2018;11:e007168.
7. Nordin S, Kozor R, Medina-Menacho K, et al. Proposed Stages of Myocardial Phenotype Development in Fabry Disease. JACC Cardiovasc Imaging 2019;12:1673-83.

8. Knott KD, Augusto JB, Nordin S, et al. Quantitative Myocardial Perfusion in Fabry Disease. Circ Cardiovasc Imaging 2019;12:e08872.

9. Scatteia A, Baritussio A, Bucciarelli-Ducci C. Strain imaging using cardiac magnetic resonance. Heart Fail Rev 2017;22:465-76.

10. Vijapurapu R, Nordin S, Baig S, et al. Global longitudinal strain, myocardial storage and hypertrophy in Fabry disease. Heart 2019;105:470-6.

11. Kawel-Boehm N, Maceira A, Valsangiacomo Buechel ER, et al. Normal values for cardiovascular magnetic resonance in adults and children. J Cardiovasc Magn Reson 2015;17:29.

12. Mathur S, Dreisbach JG, Karur GR, et al. Loss of baseto-apex circumferential strain gradient assessed by cardiovascular magnetic resonance in Fabry disease: relationship to T1 mapping, late gadolinium enhancement and hypertrophy. J Cardiovasc Magn Reson 2019;21:45.

13. Nucifora G, Muser D, Tioni C, et al. Prognostic value of myocardial deformation imaging by cardiac magnetic resonance feature-tracking in patients with a first STsegment elevation myocardial infarction. Int J Cardiol 2018;271:387-91.

14. Militaru S, Ginghina C, Popescu BA, et al. Multimodality imaging in Fabry cardiomyopathy: from early diagnosis to therapeutic targets. Eur Heart J Cardiovasc Imaging 2018;19:1313-22.

15. Biegstraaten M, Arngrímsson R, Barbey F, et al. Recommendations for initiation and cessation of enzyme replacement therapy in patients with Fabry disease: the European Fabry Working Group consensus document. Orphanet J Rare Dis 2015;10:36.

16. Takenaka T, Teraguchi H, Yoshida A, et al. Terminal stage cardiac findings in patients with cardiac Fabry disease: an electrocardiographic, echocardiographic, and autopsy study. J Cardiol 2008;51:50-9.

17. Bogarapu S, Puchalski MD, Everitt MD, et al. Novel Cardiac Magnetic Resonance Feature Tracking (CMRFT) Analysis for Detection of Myocardial Fibrosis in Pediatric Hypertrophic Cardiomyopathy. Pediatr Cardiol 2016;37:663-73.

18. Hor KN, Gottliebson WM, Carson C, et al. Comparison of magnetic resonance feature tracking for strain calculation with harmonic phase imaging analysis. JACC Cardiovasc Imaging 2010;3:144-51. 
19. Baeßler B, Schaarschmidt F, Dick A, et al. Diagnostic implications of magnetic resonance feature tracking derived myocardial strain parameters in acute myocarditis. Eur J Radiol 2016;85:218-27.

20. Bhatti S, Vallurupalli S, Ambach S, et al. Myocardial strain pattern in patients with cardiac amyloidosis secondary to multiple myeloma: a cardiac MRI feature tracking study. Int J Cardiovasc Imaging 2018;34:27-33.

21. Gruner C, Verocai F, Carasso S, et al. Systolic myocardial mechanics in patients with Anderson-Fabry disease with and without left ventricular hypertrophy and in comparison to nonobstructive hypertrophic cardiomyopathy. Echocardiography 2012;29:810-7.

22. Labombarda F, Saloux E, Milesi G, et al. Loss of base-toapex circumferential strain gradient: A specific pattern of Fabry cardiomyopathy? Echocardiography 2017;34:504-10.

23. Luetkens JA, Schlesinger-Irsch U, Kuetting DL, et al. Feature-tracking myocardial strain analysis in acute myocarditis: diagnostic value and association with myocardial oedema. Eur Radiol 2017;27:4661-71.

24. Vigneault DM, Yang E, Jensen PJ, et al. Left Ventricular Strain Is Abnormal in Preclinical and Overt Hypertrophic Cardiomyopathy: Cardiac MR Feature Tracking. Radiology 2019;290:640-8.

25. Tahir E, Starekova J, Muellerleile K, et al. Impact of Myocardial Fibrosis on Left Ventricular Function Evaluated by Feature-Tracking Myocardial Strain Cardiac Magnetic Resonance in Competitive Male Triathletes with
Normal Ejection Fraction. Circ J 2019;83:1553-62.

26. Perry R, Shah R, Saiedi M, et al. The Role of Cardiac Imaging in the Diagnosis and Management of Anderson-Fabry Disease. JACC Cardiovasc Imaging 2019;12:1230-42. Erratum in: JACC Cardiovasc Imaging. 2019 Sep;12(9):1903. doi: 10.1016/j.jcmg.2019.07.004.

27. Sado DM, White SK, Piechnik SK, et al. Identification and assessment of Anderson-Fabry disease by cardiovascular magnetic resonance noncontrast myocardial T1 mapping. Circ Cardiovasc Imaging 2013;6:392-8.

28. Pica S, Sado DM, Maestrini V, et al. Reproducibility of native myocardial T1 mapping in the assessment of Fabry disease and its role in early detection of cardiac involvement by cardiovascular magnetic resonance. J Cardiovasc Magn Reson 2014;16:99.

29. Thompson RB, Chow K, Khan A, et al. Ti mapping with cardiovascular MRI is highly sensitive for Fabry disease independent of hypertrophy and sex. Circ Cardiovasc Imaging 2013;6:637-45.

30. Liu B, Dardeer AM, Moody WE, et al. Reference ranges for three-dimensional feature tracking cardiac magnetic resonance: comparison with two-dimensional methodology and relevance of age and gender. Int J Cardiovasc Imaging 2018;34:761-75.

31. Marazzi MG, Galliera E, Vianello E, et al. Hypertension in adult Fabry's disease: is cardiotrophin-1 a diagnostic biomarker? Immun Ageing 2014;11:27.
Cite this article as: Zhao L, Zhang C, Tian J, Saiedi M, Ma C, Li N, Fang F, Ma X, Selvanayagam J. Quantification of myocardial deformation in patients with Fabry disease by cardiovascular magnetic resonance feature tracking imaging. Cardiovasc Diagn Ther 2021;11(1):91-101. doi: 10.21037/cdt20-897 